\title{
10 Jahre DAAD-Ortslektorennetz \\ Eine Geburtstagsfeier der besonderen Art
}

\author{
CHRISTOPH EHLRRS \\ Ortslektor Universität Sevilla
}

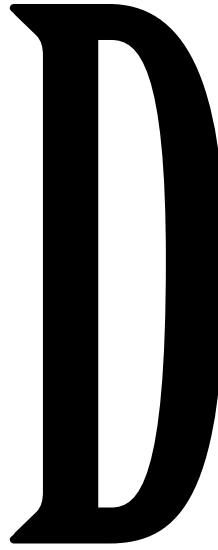

eutschland ist Großmacht, in einigen Zusammenhängen wider Willen, auf dem umkämpften 'global education market' aber sehr wohl willentlich. Für diese Art nachhaltiger Wachstumspolitik, bei der es um den Wirtschaftsstandort Deutschland, aber auch um Wissen und Werte der Höheren Bildung geht, gilt kein Understatement. Der DAAD verfügt über einen Haushalt von fast 400 Millionen Euro, getragen vor allem vom Auswärtigen Amt, dem Bundesministerium für Bildung und Forschung und dem Bundesministerium für wirtschaftliche Zusammenarbeit. Dem bescheidenen Unidozenten aus Südspanien werden diese Dimensionen schlagartig deutlich, wenn er den Hauptsitz des DAAD an der Kennedyallee in Bonn betritt: mehrere mehrstöckige, kreisverwaltungsgroße Bürogebäude reihen sich aneinander, Hunderte von Angestellten widmen sich emsig ihren Aufgaben. Wohl wenige Länder der Welt wenden solche Mittel für ihre auswärtige Bildungspolitik auf.

"In der Ferne zuhaus, mit Deutschland vernetzt". In dieser Antithese drückt der DAAD treffend den Geist seines Ortslektorennetzes aus, mit dem er die im Ausland tätigen deutschen Universitätsdozenten fördert, vernetzt und als Mittler der auswärtigen Bildungspolitik nutzt. Das Förderangebot ist groß und unsere 'alma mater', die unermüdliche Fachbereichsleiterin Elke Hanusch in der Schaltzentrale in Bonn, immer und zu jeder Zeit ansprechbar und zur Hilfe bereit (http://www.daad.de/ausland/lehren-imausland/deutsche_lehrkraefte).Siekoordiniert die 771 KollegInnen, die da momentan weltweit an der Brücke des Deutschen mitbauen: 330 in Westeuropa, etwa 40 in Nordund 31 in Südamerika, 8 in Afrika, 15 in Mittel- und Osteuropa und 384 in Asien und Ozeanien. Wie schon beim IDV, beeindruckt auch hier die internationale Projektion unserer Sprache, die den deutschen Muttersprachler ehrt und auch überrascht. So hat das Deutsche in Japan traditionell eine sehr starke Position an den dortigen Universitäten, denn zur Öffnung und Modernisierung des Landes Ende des 19.Jhs. schaute man nach den besten Modellen, und dazu gehörte die deutsche Universität und Wirtschaft. Das zweitstärkste Netz besteht in Italien. Die KollegInnen dort organisieren Fachtagungen, geben eine Zeitschrift heraus und unterstützen sich in ihren Arbeits-kämpfen, denn als ausländische Lektoren sind sie an den italienischen Universitäten vielen Schikanen ausgesetzt. Hier in Spanien sind momentan sechsundzwanzig von uns erfasst und vernetzt. Zwei Orts-lektorentreffen haben bisher stattgefunden, das erste 2008 in Sevilla, dann 2011 in Madrid, beide Male mit so erstklassigen Seminarleitern wie Karin Kleppin und Jörg Roche, und beide Male in einem sehr angenehmen und konstruktiven kollegialen Klima.

Nun war 10-jähriges Jubiläum und der DAAD hatte vom 9. bis 12. September 2011 fünfzig KollegInnen aus vierundzwanzig Ländern nach Bonn an den Rhein geladen. Für viele der Teilnehmer etwas Besonderes, denn etwa aus Australien oder Südkorea kommt man nicht so oft in die Heimat. Und die perfekte und herzliche Organisation um Elke Hanusch und Julia Kracht ließ gleich dieses großartige Gefühl aufkommen, zu Gast in der Heimat zu sein. Heimat und Ferne - um diese Konzepte webt sich auch das schmucke Sammelbändchen, das zur Gelegenheit herausgegeben und präsentiert wurde und in dem dreiundzwanzig KollegInnen aus der ganzen Welt ein kunterbuntes Kaleidoskop ihrer Erfahrungen als akademische Auswanderer bieten: In der Ferne zu Hause, die Heimat im Blick. Ortslektorinnen und Ortslektoren berichten ist ein lohnender Fundus interkultureller Erfahrungen.

Nach der Begrüßung, unter anderen durch dieDAAD-Generalsekretärin Dr.Dorothea Rühland und die Abteilungsleiterin für Germanistik und deutsche Sprache im Ausland, Dr. Gisela Schneider (man erlaube mir den Eindruck: der DAAD ist Sache vieler sehr kluger Frauen), stellten einige Ortslektoren ihre Arbeit in so verschiedenen Kontexten wie Tunesien, Tadschikistan und Südkorea vor. Aus Tunesien erzählte der Kollege sehr bewegt vom Befreiungskampf, den er aus nächster Nähe miterlebt hatte, in Tadschikistan hatte die junge Jenaer Kollegin das DaF-Institut der Aga-Khan-Universität förmlich aus dem Steppenboden gestampft, und in Südkorea nutzte der Kollege die Einsatzmöglichkeiten der allerneuesten Technologien. Und das sind nur drei der fast 800 Knotenpunkte unseres Netzes...

Anregende Redner wie Christian Bode, ehemaliger Generalsekretär des DAAD (siehe auch Interview in diesem Band) sind selten. Er hat die Universität im Kopf. In seinem Vortrag "Entwicklungen in der internationalen Hochschullandschaft im letzten Jahrzehnt - Wohin geht die Reise?” stellte er, ganz entspannt und wie aus dem Nähkästchen, Zahlen und Fakten in einen großen, auch ethischen Zusammenhang, der von Humboldt ("damals studierten an den deutschen Universitäten gerade fünftausend Privilegierte") über die wilden 


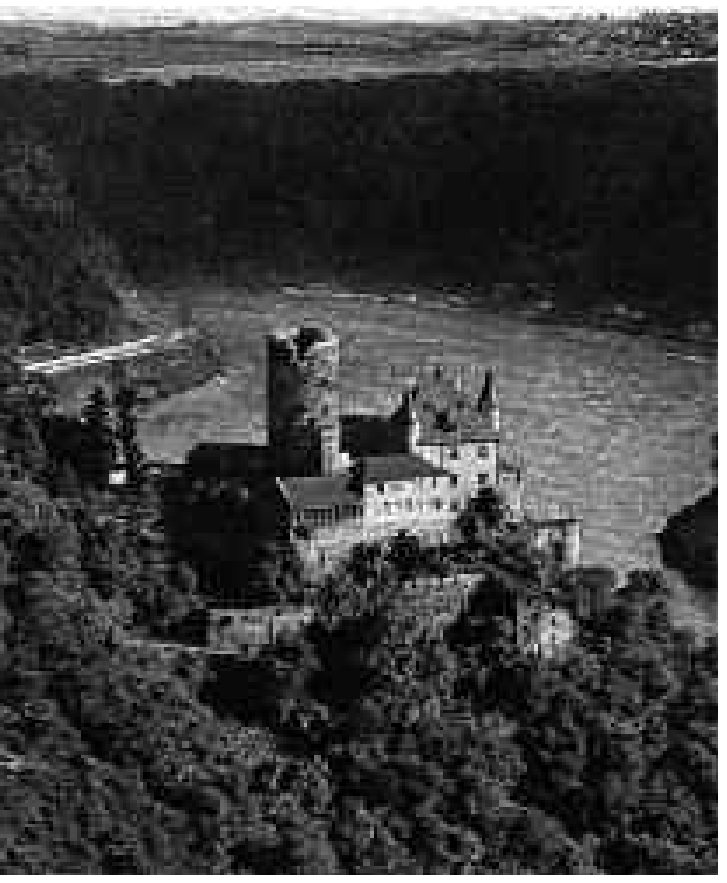

Sechziger ("Nur zwanzig Prozent Abiturienten. Und sie können sich gar nicht vorstellen, wie ungeheuer spießig Deutschland damals war") bis zu Bologna ("hat einen Kopf, aber keine Seele") und dem 'global education market' reichte. Auf diesem könne Deutschland als Bildungsstandort nur bestehen, wenn es erstens mehr Hochschulabsolventen gebe und zweitens der Ausländeranteil an ihnen erheblich stiege. Die Linie des DAAD ist klar: Die Welt ist vernetzt und wir müssen auf die Interdependenz vorbereitet sein. Deutschland müsse somit weltoffener werden, es gebe zu wenig Ausländer oder 'Bildungsausländer' (Ausländer mit deutschem Abitur) an den deutschen Hochschulen. Dabei gehe es nicht nur um Wettbewerb, sondern auch um die Lösung gemeinsamer Probleme: Erschöpfung nicht erneuer-

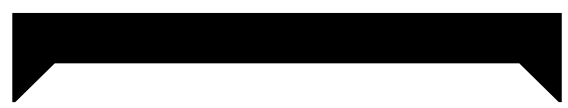

Was vor vierzig Jahren Emigranten, sind heute 'Transmigranten', die aus dem Süden Europas nachDeutschlandströmen.DieUnterschiede und Gemeinsamkeiten, Folgen und Ursachen sind Stoffgenugfür einetransnationale Arbeitsgruppe 'Kurse aus der Krise'

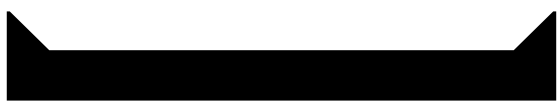

barer Ressourcen, Umweltkatastrophen etc. Die unlösbare Frage, wie der Spagat zwischen globalem (Bildungs)Kapitalismus und zunehmender globaler Armut geschafft werden soll, ließ Herr Bode allerdings auch offen. Gegenüber der Privatisierungstendenz des Bildungsmarktes trat er für mehr öffentliche Investitionen ein. Deutschland belege bei den öffentlichen Bildungsausgaben, sowohl im Verhältnis zu den öffentlichen Gesamtausgaben als auch zum BIP, einen Schlussplatz und liegt weit unter dem OCDE-Durchschnitt. Auf der anderen Seite müssen die Universitäten der Tendenz folgen und 'unternehmerischer' werden, um sich behaupten zu können.

Nach interessanten Workshops und Museumsbesuchen (die Ausstellung von Max Liebermann warf auch Licht auf ein weiteres tragisches Kapitel deutsch-jüdischer Geschichte) war am Sonntag endlich Chillen angesagt, aber standesgemäß: es ging mit dem Dampfer durch das Rheintal von Bingen nach Bonn. Deutscher geht es wohl kaum als am Deutschen Eck und seinen Burgen und Gründerzeitpalästen und Spitzendeckchen und Deutschlandfahnen in Vorgärten und Weißweingelagen zu Kaffee und Kuchen und Schiffen namens Victoria oder Patria und der Endlosschleife des Reise-Führers, der redet wie die Rheinfälle (er war natürlich Niederrheiner)... Wir glitten stundenlang wie im Traum durch die atemberaubend schöne Landschaft, und immer wieder war von den in alle Welt emigrierten KollegInnen zu hören, wie schön Deutschland doch sein könne. Es gab Zeit und Ruhe zum Quasseln - und Pläne schmieden, denn ein Ortslektor ruht bekanntlich nie. Bei Konstanz steckten die Asiaten die Köpfe zusammen und schufen ihr Video-Austauschnetz, weiter flussabwärts hoben dann die Italiener, Portugiesen und Spanier, vom nassen Element beflügelt, ihr 'Poseidon'-Projekt ans Licht der Welt, das mittlerweile auch schon mit einem Blog aufgetaucht ist (http://poseidon.podspot.de/). Wir überlegten: Was vereint uns Mediterrane außer Rotwein, Expresso, Olivenöl und dem klassischen Altertum? Richtig, die Krise natürlich. Was vor vierzig Jahren Emigranten, sind heute 'Transmigranten', die aus dem Süden Europas nach Deutschland strömen. Die Unterschiede und Gemeinsamkeiten, Folgen und Ursachen sind Stoff genug für eine transnationale Arbeitsgruppe 'Kurse aus der Krise'. Mögliche Arbeitsfelder sind die Lehre der Fachsprachen der nachgefragten Sektoren (Ingenieure, Gesundheitswesen, Dienstleistungen), die im Herkunftsland angeboten werden sollten, oder die Umstände und der unterrichtliche Umgang mit den Remigranten oder Heimgekehrten, oder die Behandlung der Krise in den deutschen Medien und Künsten aus auslandsgermanistischer Sicht.

Wir danken dem DAAD und Frau Elke Hanusch herzlichst für die schönen Tage an den Ufern unseres 'Vaters Rhein', dem wir lange den Rücken gekehrt hatten. 